\title{
Kernos
}

Revue internationale et pluridisciplinaire de religion grecque antique

20 | 2007

Varia

\section{La Banque de données sur les épiclèses divines (BDDE) du Crescam : sa philosophie}

Pierre Brulé et Sylvain Lebreton

\section{QpenEdition \\ Journals}

Édition électronique

URL : https://journals.openedition.org/kernos/189

DOI : 10.4000/kernos.189

ISSN : 2034-7871

Éditeur

Centre international d'étude de la religion grecque antique

Édition imprimée

Date de publication : 1 janvier 2007

Pagination : 217-228

ISSN : 0776-3824

Référence électronique

Pierre Brulé et Sylvain Lebreton, «La Banque de données sur les épiclèses divines (BDDE) du

Crescam : sa philosophie », Kernos [En ligne], 20 | 2007, mis en ligne le 24 mai 2011, consulté le 08 septembre 2022. URL : http://journals.openedition.org/kernos/189 ; DOI : https://doi.org/10.4000/ kernos.189 


\section{La Banque de données sur les épiclèses divines (BDDE) du Crescam : sa philosophie}

Le laboratoire Crescam de l'Université Rennes 2 met à la disposition du public une banque de données relative aux épiclèses divines (BDDE). Cette ouverture doit être accompagnée de quelques commentaires, d'éclaircissements, de justifications.

Commençons par quelques excuses. La banque est mise à disposition avec quelque retard. Dès la création de notre laboratoire nous avions annoncé notre intention de fabriquer cet outil, et c'est la même année 1997, au VIe colloque du CIERGA sur le «Panthéon des cités» que l'annonce en avait été faite1. Mais trois variables avaient été mal mesurées. Les ressources financières mises à notre disposition par le ministère de la Recherche, anémiques et tardives, nous ont obligés à n'ouvrir vraiment le chantier de la banque qu'en 2000. S'y ajoutent l'ampleur de la tâche et les limites de nos moyens humains. Malgré l'aide initiale de Nicole Belayche, qui a participé aux échanges préparatoires à la mise en marche de l'entreprise, malgré les efforts de tous ceux qui pendant toutes ces années ont œuvré à sa réalisation: Laurent Piolot (concepteur de la fichemodèle), Gwenn Quéméner, Hélène Bectarte, Céline Dubois, Pierre Tandé, Sylvain Lebreton, Yves Kernaleguen, Pierre Brulé et Jérôme Wilgaux, nous ne sommes pas aujourd'hui en mesure d'atteindre l'objectif qu'on peut assigner à une telle entreprise : l'exhaustivité (sur ce mot, nous reviendrons). Conscients par ailleurs des imperfections qu'elle présente, et que nous travaillons à éliminer, nous sollicitons votre bienveillance pour les erreurs et fautes qui peuvent subsister.

Plaidons coupable, donc. C'est qu'à l'aune des travaux préliminaires, nous avions évalué le nombre des épiclèses des "grands» dieux grecs à 200, voire 250 , et celui des dieux «mineurs » entre 50 et 80 , voire moins. Nous espérions donc pouvoir produire la BDDE à partir de 2000 enregistrements. Ce qui nous semblait raisonnable s'est avéré illusoire : le temps passant, on a successivement estimé le total à 2500 , puis 3000 , puis 3500 .. Mais c'était toujours plus : au moment où j'ai rédigé la première version de ce texte - en novembre 2005, nous en étions à 4520 entrées, alors qu'au moment où nous terminons cette

\footnotetext{
${ }^{1}$ Kernos 11 (1998), p. 16, n. 11.
} 
présentation en mars 2006, nous avons atteint le nombre «inimaginable » de $5900 \ldots$ (de sorte que nous n'osons plus pronostiquer ${ }^{2}$ ).

Pourquoi, alors, diffuser néanmoins la BDDE dans cet état ?

D'abord parce que l'incomplétude est inhérente à un tel outil. Il est à améliorer, il est toujours à compléter, il n'est jamais fini. Chaque année (chaque publication même) apporte son lot d'épiclèses nouvelles et aussi d'épiclèses déjà répertoriées mais qui apparaissent en de nouveaux lieux, dans un nouveau contexte, sans parler de nouvelles lectures de documents déjà connus.

Si, malgré son caractère partiel, nous mettons la BDDE d'ores et déjà à la disposition du public, c'est parce que la masse critique à laquelle nous sommes parvenus nous autorise à estimer qu'elle peut rendre, en l'état, de signalés services. C'est aussi en considérant que le rythme et la quantité des entrées dans la base diminue inéluctablement en raison de l'épuisement progressif des grands gisements comme telle source littéraire ou épigraphique; la tendance générale de la courbe des entrées est asymptotique, elle tend vers zéro sans l'atteindre jamais; cela justifie aussi que nous n'attendions pas.

Dans ces conditions, nous tenons donc à prévenir les utilisateurs du caractère incomplet de la BDDE, en même temps que du fait qu'il s'agit, dans l'idéal, d'un chantier en transformation constante, toujours vivant. Dans ce même projet idéal, il est souhaitable qu'un véritable dialogue s'établisse entre les utilisateurs et la gestion centrale de la base, par le biais d'une messagerie sur laquelle vous pourrez déposer critiques, corrections, interrogations, ... louanges; un lien adéquat est à votre disposition sur le site.

\section{La BDDE, sa philosophie}

L'idée-force de la constitution de cet ensemble a été la volonté d'enregistrer toutes les épiclèses du polythéisme hellénique (on verra plus loin les critères retenus pour la détermination du champ d'application de ce principe). Toutefois, si la raison d'être ultime de la BDDE est d'aider à percer à jour certains aspects du polythéisme hellénique au travers du langage des épiclèses, elle constitue immanquablement, chemin faisant, une méta-source de première importance sur la religion grecque durant un millénaire.

Mais quelle définition retenir pour l'épiclèse?

\section{Définition de l'épiclèse}

Toute détermination d'une unité de mesure comporte une part d'arbitraire, nous l'assumons.

Dans les travaux modernes, les termes 'épithète' et 'épiclèse' sont malheureusement souvent employés l'un pour l'autre et alternativement pour un même

\footnotetext{
${ }^{2}$ Au moment de boucler le volume, en juin 2007, la banque compte 6763 entrées...
} 
objet. C'est évidemment plus que fâcheux, et même intolérable lorsque l'on cherche à introduire quelque rigueur dans un classement. Si l'on prend les choses ab ovo, qu'on tente donc de partir de l'usage grec pour forger un modèle, on se heurte là aussi à des imprécisions. Les Grecs utilisent en effet aussi le

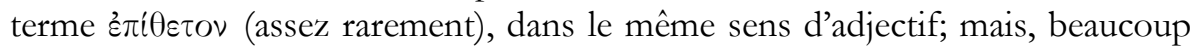
plus près de ce que nous cherchons à définir, ils ont ė $\pi \omega v u \mu i \alpha$, si près même que beaucoup d'usages du terme correspondent très exactement à ce que nous entendons par épiclèse dans la BDDE. Non le premier sens, employé pour désigner le fait de donner son nom à quelque chose, à un lieu. (On dira que le fait que Léto a délié sa ceinture (sa zônè) à tel endroit a fait donner $\tau \grave{v}$

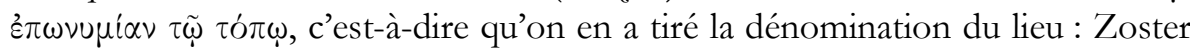
(Hypéride, fr. 64); contrairement à ce que disent les dictionnaires, très souvent, cette epônumia n'est pas à proprement parler un «surnom».) Mais le second sens, tel qu'il en est fait usage dans l'Euthydème (302c) de Platon, où l'on discourt sur ce complément déterminatif que nous nommons généralement épiclèse, et qui vient s'adjoindre comme un déterminant au nom d'un dieu :

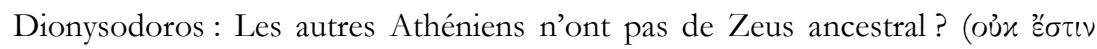
Zвù ò $\pi \alpha \tau \varrho \tilde{\varphi} O \varsigma ;)$

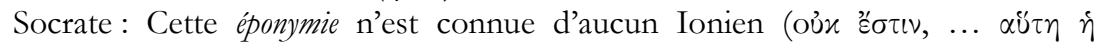

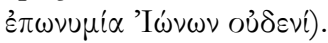

On pourrait donc très bien appeler une épiclèse une epônumia. Mais Pausanias est passé par là aussi, et c'est à lui surtout que l'on doit la vogue d'è $\pi i x \lambda \eta \sigma \iota \varsigma$ dans le sens où nous l'entendons. Lorsqu'il veut préciser sous quelle appellation tel dieu est spécifiquement honoré dans tels lieu et circonstance, c'est ce qu'il

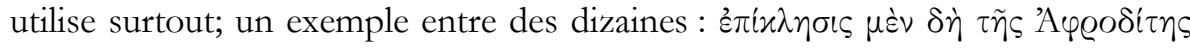

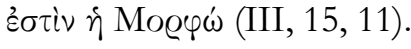

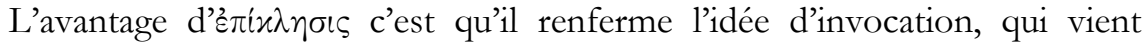

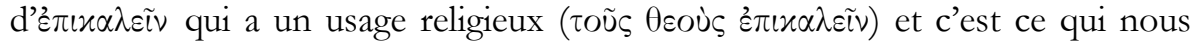
fait définitivement préférer le terme. Enfin, c'est aussi parce que l'usage des historiens, d'une part l'a consacré, et a voulu d'autre part que le terme epônumia, dans un sens en vérité restreint, désignât le fait de nommer une chose par le nom d'une autre chose ou en lui donnant celui d'un individu.

Au demeurant, dans la grande majorité des sources (la totalité des sources épigraphiques, une bonne partie des sources littéraires), les épiclèses apparaissent le plus souvent en contexte (mention d'un prêtre ou d'un sanctuaire de la divinité « épiclésée » par exemple), plutôt qu'explicitement désignées comme telles - que ce soit par epônumia ou par epikèèsis - comme c'est le cas dans les exemples que nous venons de citer. Dans la mesure où la qualité d'épiclèse n'est que rarement explicitée en contexte, son attestation revient à l'appréciation de l'interprète moderne, à chaque rédacteur de fiche en ce qui concerne la BDDE. C'est ainsi, par exemple, que la succession canonique des théonymes (théonyme + théonyme sans lien syntaxique entre eux) ne définit pas automatiquement la 
place du nom du dieu et de l'épiclèse. Ainsi, un Sarapis Dionysos peut aussi bien être un Dionysos Sarapis (même chose pour Zeus Dionysos).

Exposons et justifions donc ici les critères que nous avons suivis pour construire notre banque de données, autrement dit notre définition de l'épiclèse.

Nous définissons l'épiclèse à partir du cadre de son usage dans la nomination des puissances divines ${ }^{3}$. Dans ce polythéisme comme dans d'autres (et à un moindre degré dans certains monothéismes), les dieux sont nommés de deux façons : soit par leur nom seul (c'est le cas, dans le monde grec, dans l'épopée, dans la vulgate mythologique, dans la cité où ils sont poliades et dans les jurons), soit par un système binominal d'appellations simples qui fait coexister le nom du dieu, généralement en première position, avec un déterminant souvent adjectivé ${ }^{4}$ qui le suit - l'épiclèse - précisant une qualité particulière dudit dieu. Sanctuaires, sacrifices, dons..., les actes du culte en Grèce antique s'adressent à des dieux ainsi précisément définis ${ }^{5}$. La conséquence de cet état de ce fait, extrêmement répandu au point de pouvoir parler d'un système de désignation, c'est que pour que soit retenue une épiclèse dans notre BDDE, il convient que ce nom double désigne une entité divine recevant un culte.

Sont exclues, donc, a priori, les épithètes, les déterminants du style: 'aux lourds grondements', 'aux bras blancs', 'aux fines chevilles'... ces épithètes que l'on a pris l'habitude de qualifier de "poétiques ", puisqu'on les trouve généralement dans les textes versifiés (le vers ne fait pas la poésie, mais ce n’est pas ici

\footnotetext{
3 On résume ici les pages consacrées à cette question dans la communication de Pierre BRULÉ au VIe colloque du CIERGA « Le langage des épiclèses dans le polythéisme hellénique (l'exemple de quelques divinités féminines)», Kernos 11 (1998), p. 17-20.

${ }_{4}$ Nous avons aussi pu retenir comme épiclèses des propositions participiales, telles que

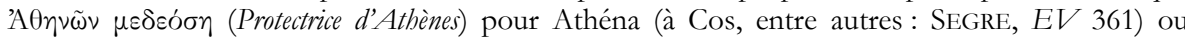
prépositionnelles, comme év xiñoıs (des jardins) pour Aphrodite (à Athènes : Pausanias, I, 19, 2 et I, 27, 3), ainsi que des noms au génitif, tels que Tí́uov (de Tiamos, le fondateur du culte, semble-til) pour Mèn (en Lydie: SEG 32, 1221 entre autres) et des noms propres (des théonymes en l'occurrence), comme Eileithyia pour Artémis (entre autres nombreux cas, à Beroia : I.Beroia, 50). Pour cette dernière catégorie, la distinction entre association et identification est souvent ténue dans les sources épigraphiques; elle tient à l'appréciation de l'éditeur (que nous n'avons pas discutée dans le cadre de la BDDE) : selon qu'il ajoute ou non une virgule entre deux théonymes, on peut être en présence d'une divinité double (nous enregistrons alors le second nom divin comme épiclèse de la première divinité nommée), ou de deux divinités associées.

${ }^{5}$ Nous touchons à la part d'arbitraire réservée plus haut. Nous savons qu'il existe des cultes où les divinités concernées sont nommées (ou peuvent être nommées) sans complément épiclétique. Les cas sont plus rares et cette absence touche certains dieux plus que d'autres. Les facteurs spatio-temporels jouent peut-être un rôle aussi. Ainsi, pour prendre une base documentaire proposant un cadre spatio-temporel large sur le thème qui nous intéresse, à savoir les trois volumes de Lois Sacrées de Fr. SOKOLOwSKI, on a, sur 32 textes concernant explicitement (les textes mentionnant seulement les noms de fêtes ou de sanctuaires mis à part) Dionysos, 19 cas où le dieu reçoit un culte sans épiclèse et 11 cas $(+2$ cas particuliers : un surnom dans un texte versifié, et une attestation indirecte) où il est épiclésé. Quoi qu'il en soit, cette façon de nommer le récipiendaire (qui peut valoir prétérition d’une épiclèse) n’invalide en rien la définition positive que nous choisissons.
} 
notre propos). Nous avons également écarté les épithètes décrivant tel type iconographique, statuaire notamment, d'une divinité. Ainsi tauromorphos, par exemple, ne peut être retenue pour épiclèse de Dionysos, car elle fait sans conteste référence à un type statuaire; la mention qu'en fait Athénée de Naucratis le laisse penser, celle de Plutarque est sans équivoque : l'épithète s'applique aux statues du dieu, et non au dieu lui-même $e^{6}$. La distinction peut toutefois dans certains cas être plus problématique ${ }^{7}$, d'autant plus que certaines épiclèses attestées en tant que telles de façon sûre peuvent faire plus ou moins directement référence à la forme de l'agalma du dieu : nous pensons ici notamment à Dionysos Orthos ${ }^{8}$ ou Dionysos Phallèn?.

Autre difficulté, celle posée par ce qu'on appelle les «surnoms» divins. Certaines épithètes, généralement "poétiques », caractéristiques de certaines divinités, peuvent en effet se substituer, par effet de synecdoque, aux théonymes génériques. C'est le cas de Phoibos ('Brillant') pour Apollon par exemple : fréquemment utilisé comme épithète du dieu dans l'Iliade et l'Odyssée (sous la forme Phoibos Apollon donc), on retrouve dans de nombreux textes poétiques dans la même Iliade - ce terme employé comme "surnom ", i.e. se substituant au théonyme Apollôn. Ce Phoibos est alors un exact équivalent d'Apollon : il n'ajoute aucune connotation, aucune coloration, si ce n'est poétique; il désigne - en théorie - la même entité divine, sans ajout ou restriction fonctionnels par rapport au théonyme générique. Le fait que, comme Apollôn, Phoibos puisse se voir attribuer des épithètes, qui peuvent être cultuelles ${ }^{10}$, semble en tout cas l'indiquer ${ }^{11}$.

De la même manière qu'une épithète poétique peut être utilisée comme surnom divin, on trouve parfois des épiclèses employées seules; le processus est semblable : si le théonyme est omis, c'est que l’identité de la divinité ne fait pas

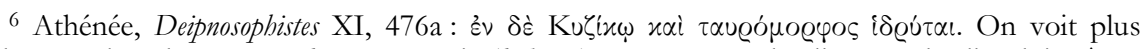
facilement dans le tauromorphos «construit (bidrutai)» une statue du dieu que le dieu lui-même;

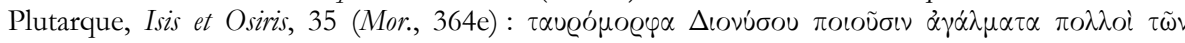
'E $\lambda \lambda \eta \eta v \omega v$; l'adjectif tauromorpha, à l'accusatif pluriel, se rapporte à agalmata et non pas à Dionusou, qui est au génitif singulier.

${ }^{7}$ C'est le cas d'une Artémis Phôsphoros étudié par L. PIOLOT, « Nom d’une Artémis! À propos de l'Artémis Phôsphoros de Messène (Pausanias IV, 31, 10) », Kernos 18 (2005), p. 113-140.

${ }^{8}$ Philochore, FGrH 328 F 5b = Athénée, Deipnosophistes II, 7, 38c-d.

9 Pausanias, X, 19, 3; cf. M. Casevitz \& Fr. Frontisi-Ducroux, «Le masque du 'Phallen'. Sur une épiclèse de Dionysos à Méthymna », RHR 206 (1989), p. 115-127.

${ }^{10}$ Une épiclèse peut bien entendu apparaittre dans un texte poétique.

11 Prenons ici pour exemple une épigramme de Lerne (IG IV, 666) mentionnant Phoibos Lukeios (1. 6); c'est Apollon Lukeios (qui reçoit un culte dans cette région) qui est ici désigné. Notons que l'épiclèse fait elle-même l'objet d'un traitement poétique, puisqu'elle est transcrite plus loin en lukoktonos (1. 8), "le tueur de loup», qui est une des différentes significations de Lukeios.
} 
de doute ${ }^{12}$. Ces deux procédés, pour proches qu'ils soient sur le plan de la forme, diffèrent cependant sur celui de la signification. En effet, le surnom se substitue au théonyme, et à lui seul, sans ajout ou restriction de sens; dans le cas de l'épiclèse employée seule, celle-ci remplace l'ensemble théonyme + épiclèse. Lorsque différentes inscriptions mentionnent le Lukeios ('Des Loups', 'Lycien') ${ }^{13}$, elles se réfèrent à l'Apollon Lukeios, dont le culte est largement répandu dans le monde $\operatorname{grec}^{14}$. Ainsi lorsque Phoibos est mentionné dans un texte, c'est à un Apollon générique qu'il est fait allusion, à l'ensemble de l'entité divine que recouvre le nom 'Apollon'; lorsque l'on rencontre Lukeios dans un texte, on est certes en présence d'Apollon, mais de cet Apollon-ci, celui qui détient ce caractère identitaire, qui jouit de cette fonction (ou ces fonctions) particulière(s) pour laquelle (lesquelles) il reçoit un culte dans une (ou plusieurs - elles sont nombreuses pour le cas présent) cité précise. L'autre différence majeure entre surnom et épiclèse tient en effet au cadre spatial dans lequel le théonyme pourra être sous-entendu, l'ensemble d'individus ayant parmi leurs références communes l'association univoque d'une divinité à un surnom ou une épiclèse. Cette différence n'est d'ailleurs pas purement spatiale, même si le cadre géographique est pour le premier cas généralement plus étendu que pour le second ${ }^{15}$; elle est plutôt fondée sur l'expérience. En effet, pour conserver notre exemple, l'ensemble des individus reconnaissant Apollon dans Phoibos, en l'occurrence la totalité du «monde grec», forme une communauté culturelle dont les références mentales communes, parmi lesquelles figurent entre autres les textes homériques, constituent ce que l'on peut appeler la « culture grecque »; l'ensemble de ceux qui voient immanquablement un Apollon dans le Lukeios forme une communauté cultuelle, généralement une cité, ou un ensemble de cités ${ }^{16}$, qui tire l'attribution univoque de cette épiclèse à ce dieu de l'expérience commune du culte rendu à ce dernier ${ }^{17}$. Nous revenons, en somme, à notre définition initiale : une épiclèse implique nécessairement un culte, une fonction et un lieu.

\footnotetext{
12 Dans le cas du nom du dieu seul dans le contexte cultuel discuté plus haut, le raisonnement peut faire le chemin inverse et imaginer que l'épiclèse correspondante allait de soi (documents épigraphiques à usage interne).

${ }^{13}$ IG IV , 557, SEG 16, 246 et 248 et SEG 17, 141 (Argos); IG IV, 872 (Épidaure); IG IV, 872 (Hermione), SEG 29, 956-958 et 965 (Métaponte); SEG 29, 391 (Sparte); IG XII 3, 389 (Théra).

${ }^{14}$ D'autres inscriptions d'Argos - pour ne prendre ici que cet exemple - mentionnent le dieu avec sa titulature complète : IG IV 559 et 658, SEG 16, 255 et SEG 17, 142.

15 Mais il y a d'importantes et d'évidentes exceptions : on pense, par exemple, aux épiclèses topographiques et «topographiques déplacées » dont un exemple est fourni par l'Ephesia, l'Artémis d'Éphèse qui a reçu un culte dans une bonne partie du monde méditerranéen antique.

16 Plusieurs cités peuvent avoir des références cultuelles communes.

17 Selon ce raisonnement, une même épiclèse employée seule pourrait correspondre à différentes divinités, selon l'endroit où l'on se trouve. Cela se vérifie pour le Iatros/Lètros qui est Asclépios à Cyrène (G. Pugliese Carrateldi, «Legge sacra di Cirene », PP 1960, p. 294-297) et Apollon à Olbia du Pont (entre autres, IGDOP 55, 65, 90), le Sôtèr qui est Asclépios à Épidaure
} 
Ce problème de frontière entre "surnoms » et épiclèses peut parfois se poser dans des dimensions toutes particulières, puisque certaines épithètes divines - pour utiliser ici un terme neutre - peuvent, d'un document à l'autre, se révéler "poétiques » (généralement employées sous la forme de surnoms) ou cultuelles. Ainsi Bakkhos ('Bacchant'), qui est à la fois bien attesté comme épiclèse ${ }^{18}$ et très fréquemment utilisé en poésie en lieu et place du théonyme Dionusos. Certes, la différence de nature entre ces deux emplois de l'épithète est, sur un plan théorique, assez nette, l'un précisant une fonction dans le cadre d'un culte, l'autre n'étant qu'un substitut littéraire; il est toutefois extrêmement malaisé, et certainement un peu malvenu, de scinder l'existence de Bakkhos - i.e. de notre point de vue, l'ensemble de ses apparitions dans les sources à notre disposition - en deux catégories tranchées, quand il n'était l'expression que d'une seule réalité, certes modulable, mais cohérente, aux yeux des Grecs. Si le surnom poétique et l'épiclèse n'ont pas pour l'historien des religions la même signification, il est difficile de n'attribuer à l'un aucune connotation fonctionnelle, et de voir cette dernière systématiquement incluse dans l'autre. Si les ensembles «Dionysos », «Bakkhos» et «activité bachique» sont largement séquents, ils ne sont toutefois pas totalement superposables: si Bakkhos renvoie dans la plupart des cas à Dionysos, il peut être associé à d'autres divinités; de plus, Bakkehos n'évoque pas nécessairement la sphère de compétence «bachique » du dieu, qui à l'inverse, peut apparaitre chez ce dernier sans que soit utilisée l'épithète qui, a priori, lui correspond. Dionysos est le «Bacchant» par excellence ${ }^{19}$; aussi, les entités désignées par le théonyme et l'épithète, fortement associées dans l'esprit des Grecs, se recoupent et peuvent s'évoquer, se sousentendre, s'inter-changer l'une et l'autre. Ce n'est pas seulement un domaine de compétence de Dionysos que le terme bakkkhos et ses dérivés recouvrent, c'est un magma constitué de fonctions divines donc, mais aussi d'attributs, de significations, un paysage religieux au sens large associé de façon privilégiée au dieu. Notons à ce propos que Bakkhos et ses dérivés, en tant qu'épiclèses peuvent être également confondus avec différents termes techniques relevant de cette sphère « bachique $»^{20}$. D'autres épithètes de Dionysos moins importantes, quantitativement s'entend, peuvent aussi être poétiques dans certains cas, cultuelles dans d'autres. C'est le cas de Lènaios ('Des Lènai', 'Du mois de

(IG IV $1^{2}, 417,438$ et 478) et à Hermione (IG IV, 1008) et Zeus à Olbia du Pont (SEG 32, 750) et à Süpü Ören en Phrygie (MAMAV, 151).

18 Dans les deux cités cariennes de Cnide ( $L S A M, 55)$ et de Tralles ( $L S A M, 75)$, pour citer deux cas parmi les mieux assurés.

${ }^{19}$ H. Jeanmaire, Dionysos. Histoire du culte de Bacchus, Paris, 1951, p.58.

20 On mentionnera ici simplement bakkhos, le «bacchant» qui, outre le dieu, peut également

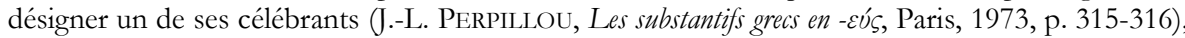
et le bakkeheion, pouvant désigner à la fois une association dionysiaque et le lieu où elle se réunit. 
Lènaiôn', 'Des Lénées') ${ }^{21}$ et de Luaios ('Libérateur')22. Cette différence d'emploi peut, là encore, être floue. En effet, au croisement de ces deux catégories d'appellations divines peuvent se trouver ce que l'on peut appeler les invocations, i.e. des surnoms, généralement au vocatif, employés dans les hymnes notamment. Leur vocation à la mise en contact avec une divinité, en la définissant éventuellement sous une certaine forme, et leur possible utilisation rituelle ne permettent-elles pas de leur attribuer une certaine portée cultuelle? La question vaut peut-être d'être posée. Quand les «femmes d'Élide» invoquent dans leur hymne Dionysos comme «Digne Taureau (axie taure) »23, c'est dans le cadre d'une fête en son honneur ${ }^{24}$, célébrant la venue du dieu avec ses

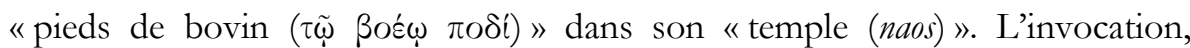
répétée deux fois, participe de la manifestation du dieu comme taureau et s'inscrit donc indéniablement dans le culte qui lui est rendu sous cette forme.

Les données lexicographiques illustrent bien d'autres difficultés qui tournent autour de la question du culte. Soit quelques gloses d'Hésychios à propos de

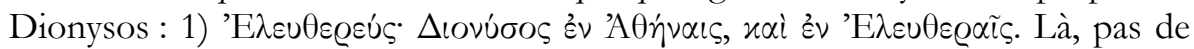
difficulté, on a affaire à une épiclèse, qui a d'ailleurs un rapport avec un culte

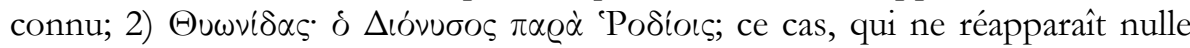
part ailleurs, ou alors sans précision de lieu (Ovide, Métamorphoses IV, 13) est fréquent: Hésychios cite souvent la cité où le nom apparait dans ses sources. S'il est peu douteux que $\Theta u \omega v i ́ \delta \alpha \varsigma$ fût une épiclèse de Dionysos à Rhodes, en l'absence de renseignements complémentaires, nous nous abstenons d'associer les épiclèses données sous cette forme à un culte; nous enregistrons la donnée; 3) Eủas $\Delta$ ıóvvбos. Doit-on retenir ce Dionysos de l'évoé ? On peut argumenter que l'évoé s'intègre dans la manifestation du contact du fidèle avec le dieu, que, compte tenu du but de la BDDE, cette touche supplémentaire au paysage dionysiaque n'est pas sans intérêt; on la retiendra " provisoirement » en arguant d'une confirmation ultérieure possible par une autre source. Nous restons toutefois conscients du caractère problématique des rapports entre le théonyme et le nom qui sert d'entrée à la glose, a fortiori si celui-ci n'est pas connu par ailleurs (contrairement à Euas, qui, avec ses «cousins » Euios et Euastèr, est un surnom bien attesté de Dionysos). Les gloses plus précises, ainsi que des recoupements avec d'autres documents (qui permettent parfois de retrouver les

\footnotetext{
21 Culte attesté en Attique (Athènes, Rhamnonte); on trouve également l'équivalent Lèneus (uniquement employé comme épiclèse) à Mykonos.

22 Attesté comme épiclèse à Éleusis. Nous ne pouvons attribuer avec autant de certitude que le fait M. Jost (Sanctuaires et cultes d'Arcadie, Paris, 1985, p. 432) de valeur fonctionnelle, et a fortiori cultuelle, au Luaios invoqué dans une épigramme de Mantinée (IG V 2, 287). Son emploi seul (sans le théonyme), au vocatif, qui plus est dans un texte versifié, nous incite plutôt à n'y voir qu'un surnom poétique.

23 Plutarque, Questions grecques, 36 (Mor., 299a-b).

${ }^{24}$ Les Thuia d'après M.P. NiLSSON, Griechische Feste von religiöser Bedeutung mit Ausscbluss der attischen, Leipzig, 1906, p. 291-292.
} 
sources utilisées par les lexicographes : ainsi le Dionysos Eleuthereus pêché par Hésychios chez Pausanias) montrent que les lexicographes peuvent tout à la fois définir par un seul théonyme une épiclèse, une épithète "poétique ", un surnom, un équivalent étranger, voire un proverbe ${ }^{25}$; il convient par conséquent d'appréhender ce type de gloses avec circonspection. Un exemple est fourni par le Dionysos Oinos (Hésychios, s.v.) : si le lien entre le dieu et le vin n'est pas discutable, sa nature, du moins telle qu'elle est exposée dans cette glose, l'est davantage: Dionysos est le vin, et le vin est Dionysos (il peut être son sang également) et c'est sans doute la portée de cette définition; mais on ne peut exclure la possibilité d'une épiclèse (ou d'une épithète, d'un surnom, etc.) Oinos de Dionysos - qui ne contredit d'ailleurs en rien une possible définition théorique - mentionnée dans une source disparue. Sans compter les gloses énigmatiques, tant sur le plan de la signification du terme défini que de la portée de la définition, telle celle de la Souda, s.v. 'A $\varphi \theta \alpha \dot{\alpha}$ ' $\Delta$ ıóvvбos.

Dans sa rigueur, une telle définition de l'épiclèse (épithète - ou autre donc formant avec le théonyme un nom double désignant une entité divine recevant un culte) pose également des problèmes documentaires. Dans de très nombreux cas nous sommes dans l'impossibilité d'assurer que l'occurrence du nom double doive être interprétée comme le témoignage d'un culte. D'abord parce que cela dépend de l'extension que l'on donne à ce mot. De l'existence d'une dédicace - surtout sur un support dont on ne détermine pas forcément la nature - nous ne pouvons restituer celle d'un «culte», avec ce que nous pouvons mettre dans ce terme de règles liturgiques, de dimension collective... Néanmoins, ce pauvre texte témoigne bien d'un acte de dévotion à une parcelle du divin hellénique, et cela nous suffit. Le culte, cela peut être modeste et individuel. La définition (et l'on voit bien pourquoi on évoquait plus haut l'arbitraire) s'élargit donc aux expressions du contact recherché par les hommes auprès du divin.

C'est qu'en effet nous ne perdons pas de vue que ce sont les dieux grecs et le polythéisme qui sont, au-delà de la BDDE-méta-texte, le sujet ultime de l'enquête, non le(s) culte(s). Nous avons néanmoins voulu que soient enregistrés deux outils permettant, dans la masse items, de distinguer ceux qui peuvent ressortir de la catégorie du cultuel. Ce sont les rubriques consacrées l'une à la nature du support des textes épigraphiques, l'autre, d'une façon plus générale, interroge le contexte (lieu, autres divinités citées, texte lui-même) pour conclure par oui ou par non à l'existence avérée d'un culte de cette divinité. C'est au rédacteur de la fiche d'en juger. Tous ces cas litigieux - inévitables dans une

\footnotetext{
25 Pour n’exposer ici que le cas le plus éloigné de l'épiclèse, mentionnons la glose é@eßív $\theta \imath v o \varsigma$ $\Delta$ ıóvvoos (Hesychios s.v., repris par Photius, Lexique s.v. et Souda, s.v.) qui précise qu'il s'agit là d'un proverbe que l'on peut traduire par « des prunes » i.e. "sans valeur ». On peut supposer, en théorie, que d'autres gloses moins «bavardes» peuvent mentionner de semblables expressions comprenant un théonyme, sans préciser leur nature.
} 
démarche qui, telle que la nôtre, vise à la fois à l'exhaustivité et à l'objectivité, auxquels se heurte une définition de l'épiclèse restée trop théorique, font que, dans la pratique, nous avons le plus souvent, dans le doute, préféré enregistrer dans la BDDE des épiclèses « imparfaites » plutôt que de les écarter.

\section{Les dieux et l'au-delà polythéistique}

La géographie contemporaine fait du changement d'échelle l'un de ses outils les plus performants pour interroger l'espace; il est aussi l'idée-force qui guide notre projet, son principe fondamental. S'agissant du panthéon (ou des...), il est en effet temps d'offrir à la recherche, grâce à un changement de focale, une triple modification des points de vue.

\section{Collection(s)}

Plaçons-nous d'abord dans la perspective de l'étude d'une divinité singulière.

La BDDE offre la possibilité de saisir (non pas forcément d'un coup d'œil, pour certaines divinités qui s'offrent des centaines d'épiclèses, mais au moins bien plus aisément) l'ensemble de son "paysage épiclétique ». Cette perspective d'enquêtes systématiques permet donc de rompre avec l'attitude «dilettante » qui consiste, pour l'exégète, à faire son propre chemin, arbitraire, parmi les épiclèses de la divinité, soit en délaissant celles qui pourraient se trouver hors d'un itinéraire préconçu (conscient ou non) soit, simplement, par ignorance. Il s'agit de donner la possibilité au chercheur de passer de l'échantillon, même lorsqu'il est paradigmatique, à la collection, lui offrant ainsi ce changement d'échelle générateur de lectures nouvelles (dont celle qui consiste à aller et venir de la collection à l'échantillon).

Si l'usage de la BDDE est justifié pour l'étude d'un dieu, il l'est plus encore quand il s'agit de passer à l'échelon supérieur, à l'au-delà du polythéisme; le polythéisme compris comme un organisme certes segmenté en individuations divines, mais que, précisément, les épiclèses, par la constitution de champs sémantiques cohérents (ainsi celles, ou les familles sémantiques de celles, qui s'appliquent à divers dieux), contribuent à rendre compact. Un des enseignements de la BDDE, c'est que, si le "paysage épiclétique» compose une «forme » et une «couleur» particulières pour chaque dieu - l'idée est banale, mais le résultat est en partie renouvelé -, un changement radical d'échelle, effaçant l'horizon premier et «naturel » des dieux pour passer à l'au-delà du polythéisme "global», permet de s'apercevoir qu'il existe des formes et des couleurs communes à l'ensemble de ces paysages panthéoniques, et que ce sont les épiclèses ${ }^{26}$ qui, prises individuellement ou regroupées par familles sémantiques, disent ce divin d'au-delà (et d'en deçà) des dieux. Autrement dit, la

${ }^{26}$ Pas seulement elles, bien sûr. 
collection des épiclèses dit plus et mieux le polythéisme de tel endroit et de telle époque qu'elle ne dit les dieux.

De telles virtualités permettent de tracer deux perspectives générales à la recherche.

Des études croisées. Il s'agit surtout de mettre en évidence les rapports d'identité et de différence entre les divinités. L'étude des champs sémantiques communs aussi bien celle des champs exclusifs les uns des autres pourra être menée de façon systématique par la simple fonction de tri ouverte par le logiciel.

Des études globales. D'une façon apparemment paradoxale, cette BDDE, qui illustre plus que toute autre perspective le foisonnement inouï du polythéisme par le rassemblement des épiclèses de l'ensemble des dieux, permet de revenir sur la question de la définition $d u$ dieu. Comme si les définitions partielles de chacun permettaient de brosser un portrait robot de ce que c'est qu'une divinité.

\section{Histoire}

Nous avons décidé de ne donner aucune limite chronologique dans l'établissement de la BDDE : des premières apparitions des épiclèses à leur disparition (du VIII ${ }^{e}$ av. J.-C. ${ }^{27}$ au IV e s. ap.) et, évidemment, de nous astreindre à dater les occurrences. Là encore, on peut se placer à deux niveaux : le micro ou le méga. De façon élémentaire, on peut dater l'apparition d'une épiclèse, ici ou là, pour telle divinité (faut-il dire que la date de telle occurrence ne donne pas celle de l'«apparition» de telle épiclèse, mais celle de son premier enregistrement?), cela permet aussi de comparer cette date avec celle de la même épiclèse pour d'autres divinités (penser à sôter et dérivés), ce qui peut être un outil d'étude de l'histoire des vertus «panthéoniques » de telle épiclèse. En changeant la focale, on peut tenter de mesurer ce qu'on nous excusera d'appeler le «mouvement épiclétique ». En effet, un examen même rapide de la BDDE dans sa perspective historique révèle très vite une dimension que l'absence de collection classée interdisait de voir en pleine lumière : le temps constitue un facteur fondamental de l'évolution quantitative des épiclèses. Du IV au I ${ }^{\text {er }}$ siècle av. J.-C., le nombre des épiclèses augmente spectaculairement, certaines plus que d'autres, on voit se répandre quelque chose qui ressemble à des modes - reste évidemment à gommer autant que possible ce que peut avoir de trompeur l'effet documentaire. Bref, malgré le nombre trop important d'occurrences difficiles à dater, de nouvelles exégèses s'ouvrent dans cette dimension parce que (tant pis pour le truisme) le polythéisme évolue; la BDDE constitue pour cela un instrument de mesure.

\footnotetext{
${ }^{27}$ Et même avant. Ce cadre chronologique initialement retenu a été élargi avec l'introduction d'épiclèses d'époque mycénienne (certes très peu nombreuses) tirées de textes en linéaire B.
} 


\section{Géographie}

L'insuffisance de nos ressources matérielles nous a malheureusement contraints à abandonner une partie du projet initial qui devait couvrir aussi les autres polythéismes antiques. Nous avons donc concentré notre attention sur le polythéisme grec. La définition de l'aire prise en compte n'a donc pas dépendu de la géographie, mais de la culture, c'est-à-dire, en définitive, de la langue (nous n'avons enregistré que les sources en $\left.\operatorname{grec}^{28}\right)$. L'espace couvert est donc constitué de la partie hellénophone du monde antique dans ses définitions historiques successives. Les conséquences complexes des contacts de culture qui ont transformé le panthéon hellénique classique se lisent clairement à travers une utilisation chronologique de la BDDE. C'est ainsi que, jouant des variables historiques et géographiques, non seulement au plan quantitatif, mais aussi au plan qualitatif, l'Anatolie et le Proche-Orient voient croître leur position relative. Mais, dans ce domaine comme dans le précédent, on doit se garder de mesurer l'importance des transformations sans tenir compte de l'effet trompeur des variations quantitatives de la documentation.

Tout en sachant que nombre d'occurrences ne sont pas localisées ou ne le sont qu'approximativement, de la même façon que l'enregistrement de la date des occurrences devrait déboucher sur des lectures diachroniques du panthéon hellénique, la $B D D E$, en permettant de localiser leur provenance au double plan local et régional, ouvrira la possibilité de lectures spatiales des épiclèses : comme les dieux et avec eux, les épiclèses « se plaisent » ici ou là, dans telle cité, bien sûr, mais aussi dans telle région ou ensemble de régions; en utilisant les fonctions de tri du logiciel, en changeant d'échelle, par cité, par région, en affinant ensuite, éventuellement, par période, il sera possible d'étudier les « mouvements épiclétiques».

On peut juger à cette courte présentation que nous avons appliqué un maître mot à l'entreprise : étendre. Étendre l'enquête, le temps, l'espace, toutes ces variables à la fois ou l'une d'entre elles, la BDDE permet tous ces jeux ${ }^{29}$.

Pierre BRULÉ

Sylvain LEBRETON

Crescam - Université de Rennes 2

novembre 2006

Courriels:slyouse@yaboo.fr pierre.brule@numericable.fr

\footnotetext{
${ }^{28}$ Nous n'avons enregistré d'épiclèses écrites dans une autre langue que le grec que lorsqu'elles traduisaient manifestement une épiclèse d'origine grecque (ainsi tel auteur romain « traduisant » une épiclèse).

${ }^{29}$ Ce qu'on peut appeler le «mode d'emploi » de la banque est expliqué par un lien sur le site (http://www.uhb.fr/sc_sociales/crescam/) ou cliquer sur le site du Crescam (http://www. crescam.net /modules/icontent/index).
} 